Artigo Original Original Article

\author{
Lívia Rodrigues Santos ${ }^{1}$ \\ Letícia Almeida $^{1}$ \\ Letícia Caldas Teixeira² \\ lara Bassi $i^{3}$ \\ Ada Ávila Assunçãa ${ }^{4}$ \\ Ana Cristina Côrtes Gama²
}

Descritores

Voz

Docentes

Fonoterapia

Adesão do paciente

Disfonia

Fonoaudiologia

Keywords

Voice

Faculty

Speech therapy

Patient compliance

Dysphonia

Speech

Language and hearing sciences

Endereço para correspondência:

Lívia Rodrigues Santos

Av. Alfredo Balena, 190/275,

Santa Efigênia, Belo Horizonte (MG),

Brasil, CEP: 30130-100.

E-mail: livia4_fono@hotmail.com

Recebido em: 18/1/2012

Aceito em: 16/8/2012

\section{Adesão das professoras disfônicas \\ ao tratamento fonoterápico}

\section{Adherence of the dysphonic teachers in speech therapy}

\section{RESUMO}

Objetivo: Estudar os possíveis fatores associados à adesão ao tratamento fonoaudiológico para a disfonia, em mulheres professoras da Rede Municipal de Ensino de Belo Horizonte. Métodos: Trata-se de um estudo retrospectivo, no qual foram analisados 251 prontuários de professores atendidos no Ambulatório de Voz do Serviço de Fonoaudiologia do Hospital das Clínicas da Universidade Federal de Minas Gerais (AV-UFMG) no período de 2007 a 2009. Foram coletados os seguintes dados: idade, número de sessões programadas, número de faltas, número de filhos, tipo de disfonia, grau da disfonia, renda mensal familiar e escolaridade. Calculou-se a distância, em quilômetros, da residência ao local do tratamento e do local de trabalho ao local do tratamento fonoaudiológico. Analisou-se também o Índice de Vulnerabilidade Social do local de trabalho. Resultados: Dos 251 prontuários analisados, 135 preencheram os critérios de inclusão. Destes $89(65,93 \%)$ correspondem ao grupo que recebeu alta e $46(34,07 \%)$ ao grupo que abandonou o tratamento. Das variáveis estudadas, apenas o número de faltas e o tipo de disfonia apresentaram relação com a adesão à terapia de voz. Conclusão: O número de ausências às sessões, fator relacionado ao tratamento, e a disfonia do tipo organofuncional, aspecto referente ao quadro clínico, mostraram-se associados ao abandono da terapia de voz.

\section{ABSTRACT}

Purpose: To study the possible factors associated with adherence to the speech-language pathology treatment of dysphonia in female teachers of municipal schools of Belo Horizonte. Methods: This was a retrospective study that analyzed the medical records of 251 teachers treated at the Voice Ambulatory Speech Language Pathology Service, Hospital das Clínicas da Universidade Federal de Minas Gerais (AV-UFMG) from August 2007 to December 2009. The collected data included age, number of scheduled sessions, number of absences, number of children, type of dysphonia, degree of dysphonia, monthly family income, and education. We calculated the distance, in kilometers, of the residence to the place of treatment and the workplace to place of treatment. We also analyzed the social vulnerability index of the workplace. Results: The results of this research indicated that of the 135 records that were included in the study, 89 (65.93\%) corresponded to the discharged group and 46 (34.07\%) corresponded to the abandonment group. In comparison with all the variables studied, only the number of faults and the type of dysphonia were associated with poor adherence to voice therapy. Conclusion: Results showed that the number of absences in sessions, treatment-related factor, organofuncional dysphonia type, and clinical-related factor were all associated with the abandonment of voice therapy.

Trabalho realizado no Curso de Fonoaudiologia, Universidade Federal de Minas Gerais - UFMG - Belo Horizonte (MG), Brasil.

(1) Curso de Fonoaudiologia, Universidade Federal de Minas Gerais - UFMG - Belo Horizonte (MG), Brasil. (2) Departamento de Fonoaudiologia, Faculdade de Medicina, Universidade Federal de Minas Gerais UFMG - Belo Horizonte (MG), Brasil.

(3) Programa de Pós-Graduação (Doutorado) em Saúde Pública, Departamento de Medicina Preventiva e Social, Universidade Federal de Minas Gerais - UFMG - Belo Horizonte (MG), Brasil.

(4) Departamento de Medicina Preventiva e Social, Faculdade de Medicina, Universidade Federal de Minas Gerais - UFMG - Belo Horizonte (MG), Brasil.

Conflito de interesse: nada a declarar. 


\section{INTRODUÇÃO}

Disfonia é qualquer alteração da voz decorrente de um distúrbio funcional e/ou orgânico da laringe e/ou do trato vocal. Pode estar associada a inúmeros sintomas, como cansaço ou esforço ao falar, rouquidão, afonia, intermitência na sonoridade, falta de projeção vocal, entre outros ${ }^{(1)}$.

Os professores, considerados profissionais da voz, fazem parte de um grupo de risco para o desenvolvimento de tais alterações, como mostram os dados de licenças médicas, afastamentos e readaptações funcionais ${ }^{(2,3)}$. As implicações das disfonias para o desempenho do exercício profissional encontram-se bem documentadas ${ }^{(4)}$. Porém, sabe-se que o impacto das disfonias em professores não se restringe à esfera individual, uma vez que as repercussões laborais citadas se manifestam em escala social, econômica e profissional, tendo em vista o ofício desses docentes ${ }^{(5)}$.

As disfonias podem ser tratadas por meio da fonoterapia cujo foco é a mudança comportamental e, por essa razão, depende da participação ativa do paciente em todo o processo. Assim, é crucial que o paciente se aproprie do plano terapêutico ${ }^{(6,7)}$.

Mesmo em vigência de sintomas vocais e de suas consequências negativas sobre a qualidade de $\operatorname{vida}^{(8)}$, reconhece-se que a adesão à fonoterapia não é imediata(7), não sendo raro o abandono do tratamento. A fraca adesão aos tratamentos de longa duração é um fenômeno mundial, como se vê nos casos de doenças crônicas ${ }^{(9)}$. Diversos fatores estão imbricados no comportamento de adesão e dizem respeito às características do profissional, ao tipo e à gravidade dos sintomas, à complexidade do tratamento, aos aspectos psicológicos, culturais e socioeconômicos dos pacientes ${ }^{(10,11)}$.

A adesão é um processo dinâmico, influenciado por uma série de fatores que predispõem ou não à continuidade do tratamento, incitando a estratégia de monitoramento contínuo ${ }^{(12)}$. Estudos sobre a adesão à terapia de voz apontam que a maioria dos pacientes não concluem a fonoterapia apesar da reconhecida efetividade dos recursos terapêuticos atuais ${ }^{(13,14)}$.

Sob esse prisma, assumem relevância os projetos investigativos que buscam identificar e compreender as mediações que concorrem para a adesão ao tratamento fonoaudiológico das disfonias. Compreender os fatores associados à adesão à fonoterapia pelos professores pode auxiliar na criação/ adoção de estratégias capazes de otimizar o tratamento e, consequentemente, de reduzir os prejuízos laborais decorrentes da disfonia. Dessa forma, a presente pesquisa teve o objetivo de estudar os possíveis fatores associados à adesão ao tratamento fonoaudiológico para a disfonia, em professoras da Rede Municipal de Ensino de Belo Horizonte (RME-BH).

\section{MÉTODOS}

Trata-se de uma pesquisa retrospectiva, observacional e analítica. Foram analisados os prontuários de 251 professores disfônicos da RME-BH, que se submeteram à terapia fonoaudiológica no Ambulatório de Voz do Serviço de Fonoaudiologia do Hospital das Clínicas da Universidade
Federal de Minas Gerais (AV-UFMG). Os professores da RME-BH são periodicamente submetidos a uma avaliação pelas fonoaudiólogas da Gerência de Saúde e Perícia Médica (GSPM). Diante da necessidade de tratamento ou de esclarecimento sobre a fisiologia da produção vocal e cuidados com a voz, eles são encaminhados ao ambulatório de voz da instituição. Ressalta-se que o total de prontuários analisados foi determinado pelo número de pacientes atendidos no ambulatório de voz da instituição no período de agosto/2007 a dezembro/2009.

Foram adotados os seguintes critérios de inclusão: ser professor, do gênero feminino, com disfonia; ter recebido encaminhamento da GSPM da Prefeitura de Belo Horizonte; e possuir documentação clínica completa, incluindo a avaliação fonoaudiológica e otorrinolaringológica. Como critérios de exclusão, foram adotados: apresentar queixa fonoaudiológica não relacionada à voz; ter comparecido apenas à avaliação fonoaudiológica ou às oficinas de orientação vocal (três encontros cuja finalidade é abordar temas relativos à produção vocal e fornecer orientações sobre o comportamento vocal na prática profissional). Optou-se por excluir professores do gênero masculino devido ao reduzido número de homens na docência.

Considerou-se como variável resposta, ou seja, a variável de maior interesse da pesquisa, o status de "alta" ou "abandono" da terapia fonoaudiológica. Os critérios de alta fonoaudiológica utilizados no ambulatório de voz da instituição são os seguintes: ausência de queixa de fadiga vocal, qualidade vocal adaptada às condições anatomofuncionais da laringe (avaliada de forma perceptivo-auditiva e por meio de exames otorrinolaringológicos) e/ou adequação da resistência vocal à demanda social e profissional do paciente. Considera-se situação de abandono do tratamento: três faltas consecutivas não justificadas, comunicação da impossibilidade de comparecimento ou opção declarada pela interrupção do tratamento.

Do conjunto de 251 prontuários selecionados previamente para o estudo, 135 (53,78\%) foram incluídos e 116 (46,22\%) excluídos (Figura 1). Os prontuários excluídos representavam: 14 (5,58\%) homens; $74(29,48 \%)$ casos devido à incompletude dos dados de prontuário, dos quais $38(15,14 \%)$ eram pertencentes ao grupo que recebeu alta e $36(14,34 \%)$ eram pertencentes ao grupo que abandonou o tratamento; $11(4,38 \%)$ professoras que participaram apenas das oficinas de orientação vocal, sem indicação de tratamento fonoaudiológico; e 17 (6,77\%) professoras que não compareceram à sessão seguinte à avaliação.

Os seguintes dados foram coletados dos prontuários: idade, número de sessões programadas, número de faltas (categorizado pela mediana), número de filhos, tipo de disfonia ${ }^{(15)}$, grau da disfonia $^{(16)}$ (indicado pelo parâmetro G da escala GRBASI), renda mensal familiar e escolaridade.

Calculou-se a distância, em quilômetros, da residência da professora ao local do tratamento e do local de trabalho ao local do tratamento fonoaudiológico. Analisou-se também o Índice de Vulnerabilidade Social (IVS) da escola de inserção da professora, a fim de apreender elementos do contexto. No conjunto, esses dados foram considerados variáveis explicativas na análise. 


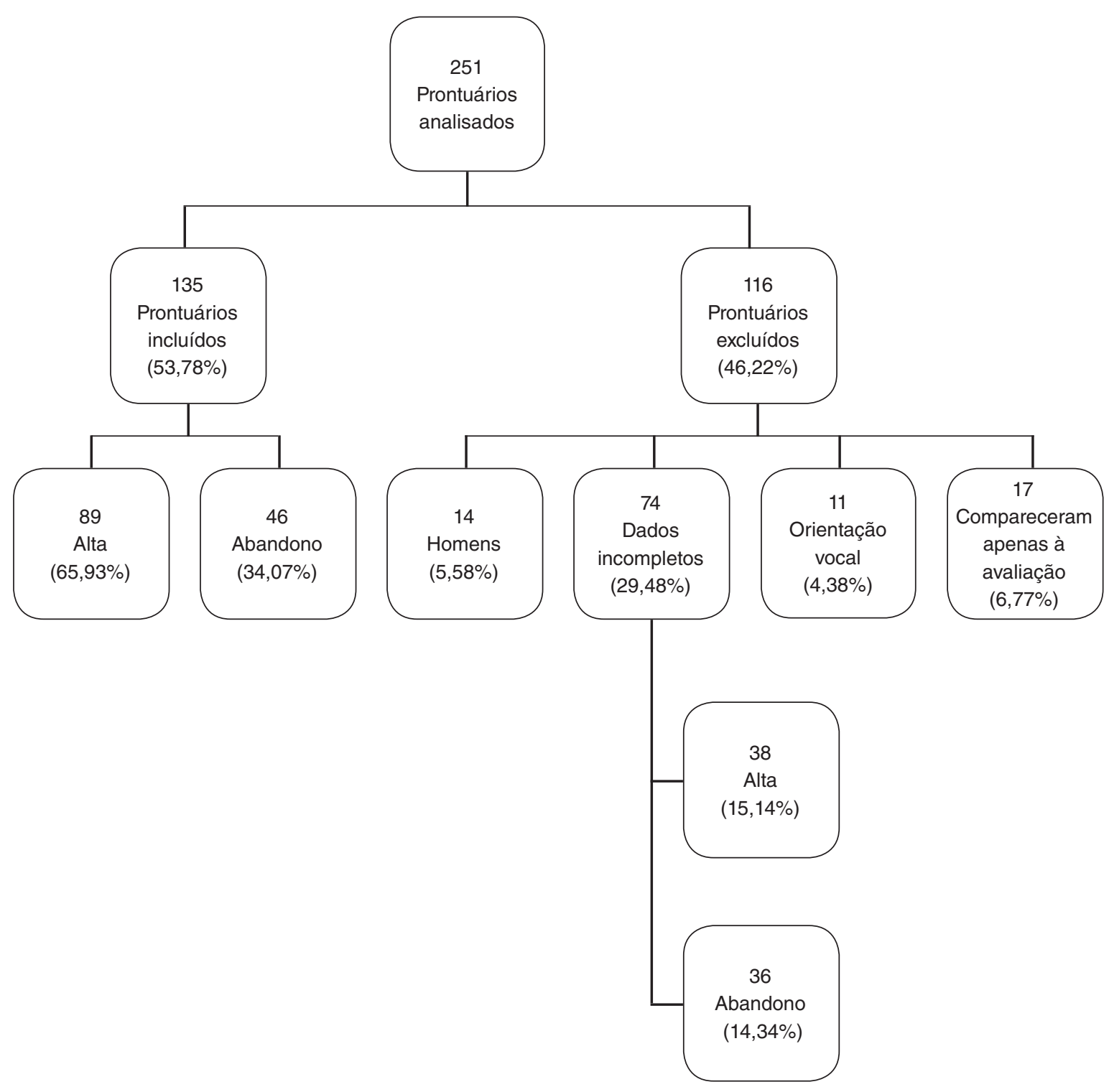

Figura 1. Diagrama representativo da distribuição da amostra

Para a análise estatística utilizou-se o programa SPSS (Statistical Package for the Social Sciences), versão 18.0. O procedimento de análise incluiu a descritiva dos dados por meio de medidas de tendência central, dispersão, medidas absolutas e porcentagens. Posteriormente para a análise dos fatores associados à variável alta e abandono foi utilizada a Regressão Logística Binária. A magnitude da associação de cada fator com o abandono da terapia foi aferida pelo Odds Ratio e a significância estatística pelo intervalo de confiança (IC) de 95\%.Todos os fatores associados ao nível de $p \leq 0,20$ na análise univariada foram incluídos no modelo multivariado por meio de deleção sequencial de variáveis. O modelo final multivariado contemplou as variáveis associadas ao nível de $p \leq 0,05$.

Consideramos o nível de significância de $\mathrm{p}<0,20$ porque esta etapa da análise univariada serve como critério de seleção das variáveis que entrarão em um modelo final da pesquisa. Como se trata de uma etapa inicial e não definitiva da análise de dados, podemos ser menos rigorosos e adotar níveis de significância maiores que o usual (por exemplo, 0,15 ou 0,20), para não correr o risco de desprezar variáveis importantes. $\mathrm{O}$ teste de ajuste do modelo Hosmer-Lemeshow foi utilizado para a verificação do modelo final.

A coleta de dados se deu no AV-UFMG. A pesquisa foi aprovada pelo Comitê de Ética em Pesquisa da mesma instituição, sob o parecer ETIC 482/08. Todos os participantes assinaram o Termo de Consentimento Livre e Esclarecido.

\section{RESULTADOS}

Procedeu-se à categorização dos sujeitos incluídos em dois grupos, alta ou abandono, 89 (65,93\%) e 46 (34,07\%), respectivamente. As variáveis contínuas utilizadas na pesquisa foram descritas por meio de medidas centrais e de dispersão (Tabela 1). A média de idade foi de 41,74 anos para o grupo 
Tabela 1. Descrição das variáveis contínuas estudadas nos grupos de pacientes que receberam alta e abandonaram o tratamento

\begin{tabular}{lccc}
\hline Variável & Situação & Média & DP \\
\hline \multirow{2}{*}{ Idade } & Abandono & 41,74 & 7,63 \\
& Alta & 40,16 & 8,83 \\
Número de filhos & Abandono & 1,15 & 1,05 \\
& Alta & 1,06 & 1,04 \\
Número de sessões & Abandono & 15,85 & 7,94 \\
programadas & Alta & 15,03 & 7,59 \\
Distância & Abandono & 13,54 & 7,01 \\
residência- terapia & Alta & 15,63 & 10,57 \\
(km) & Abandono & 16,29 & 6,19 \\
Distância trabalho- & Alta & 15,33 & 6,46 \\
terapia $(\mathrm{km})$ & &
\end{tabular}

Legenda: DP = desvio-padrão

abandono (DP 7,63) e 40,16 para o grupo alta (DP 8,83). Quanto ao número de filhos e o número de sessões programadas, maiores médias foram obtidas pelo grupo abandono. $\mathrm{O}$ grupo alta apresentou maior média para a distância entre a residência e o local de terapia. Já a distância entre o local de trabalho e o local de terapia teve média semelhante nos dois grupos.

$\mathrm{Na}$ análise univariada, a associação das variáveis descritas acima com o grupo alta/abandono não foi significativa ao nível de $\mathrm{p}<0,20$, a saber: idade ( $\mathrm{p}=0,302)$, número de filhos ( $\mathrm{p}=0,610)$, número de sessões programadas $(\mathrm{p}=0,250)$, distância residência-terapia $(\mathrm{p}=0,228)$ e distância trabalho-terapia $(\mathrm{p}=0,405)$.

Foram obtidas, ainda, as proporções das variáveis categóricas (Tabela 2). O grupo abandono foi caracterizado por

Tabela 2. Descrição das variáveis categóricas estudadas nos grupos de pacientes que receberam alta e abandonaram o tratamento

\begin{tabular}{|c|c|c|}
\hline \multirow{2}{*}{ Variável } & Abandono & Alta \\
\hline & $\mathrm{n}(\%)$ & $\mathrm{n}(\%)$ \\
\hline \multicolumn{3}{|l|}{ Faltas } \\
\hline Até 2 faltas & $16(34,8)$ & $61(68,5)$ \\
\hline 3 ou mais faltas & $30(65,2)$ & $28(31,5)$ \\
\hline \multicolumn{3}{|l|}{ Grau da disfonia } \\
\hline Normal & $3(6,5)$ & $6(6,7)$ \\
\hline Leve & $28(60,9)$ & $59(66,3)$ \\
\hline Moderado/severo & $15(32,6)$ & $24(27,0)$ \\
\hline \multicolumn{3}{|l|}{ Tipo de disfonia } \\
\hline Funcional & $10(21,7)$ & $39(43,8)$ \\
\hline Organofuncional & $36(78,3)$ & $50(56,2)$ \\
\hline \multicolumn{3}{|l|}{ Renda Familiar } \\
\hline Acima de $\mathrm{R} \$ 2400,00$ & $22(47,8)$ & $30(33,7)$ \\
\hline$R \$ 1600,00-2400,00$ & $15(32,6)$ & $40(44,9)$ \\
\hline $\mathrm{R} \$ 960,00-1600,00$ & $9(19,6)$ & $19(21,3)$ \\
\hline \multicolumn{3}{|l|}{ Escolaridade } \\
\hline Pós-graduação & $19(41,3)$ & $44(49,4)$ \\
\hline Até superior & $27(58,7)$ & $45(50,6)$ \\
\hline \multicolumn{3}{|l|}{ IVS - local de trabalho } \\
\hline Acima de 0,63 & $16(34,8)$ & $19(21,3)$ \\
\hline 0,5 a 0,63 & $18(39,1)$ & $49(55,1)$ \\
\hline 0,4 a 0,5 & $11(23,9)$ & $13(14,6)$ \\
\hline 0,28 a 0,4 & $1(2,2)$ & $4(4,5)$ \\
\hline Até 0,28 & $0(0)$ & $4(4,5)$ \\
\hline
\end{tabular}

Legenda: IVS = índice de vulnerabilidade social apresentar $65,2 \%$ dos participantes com três ou mais faltas ao tratamento fonoaudiológico, grau de disfonia leve, tipo de disfonia organofuncional e renda familiar entre $\mathrm{R} \$ 1600,00$ e R $\$ 2400,00$. Os participantes do grupo abandono possuem formação acadêmica até o nível superior e a maioria dos pacientes trabalhava em um local cujo IVS foi acima de $0,63 \mathrm{ou}$ IVS entre 0,5 e 0,63 .

$\mathrm{Na}$ análise univariada, as associações das variáveis referentes à escolaridade, grau de disfonia, renda familiar e IVS com o grupo alta/abandono não foram significativas ao nível de $\mathrm{p}<0,20$, a saber: escolaridade $(\mathrm{p}=0,370)$, grau de disfonia leve $(\mathrm{p}=0,775)$ grau de disfonia moderado/severo $(\mathrm{p}=0,493)$, renda familiar $\mathrm{R} \$ 1.600,00-2.400,00(\mathrm{p}=0,375)$, renda familiar R\$ 960,00-1.600,00 ( $\mathrm{p}=0,644)$, IVS 0,5-0,63 ( $\mathrm{p}=0,999)$, IVS 0,4-0,5 ( $\mathrm{p}=0,999)$, IVS $0,28-0,4(\mathrm{p}=0,999)$ e IVS até 0,28 $(\mathrm{p}=0,999)$. As associações das variáveis "faltas" e "tipo de disfonia" com o grupo alta/abandono foram significativas ao nível de $p<0,20$, a saber: faltas $(p<0,000)$ e tipo de disfonia $(p=0,013)$.

Foi apresentado o modelo final multivariado com as variáveis que foram estatisticamente associadas ao grupo alta/abandono ao nível de significância p $<0,05$ (Tabela 3). Apresentou-se a estatística odds ratio (OR) ou a probabilidade de ocorrência do evento. As variáveis referentes ao número de faltas e ao tipo de disfonia foram associadas ao abandono da terapia. Pacientes com três ou mais faltas tiveram 3,67 mais chances de abandonar o tratamento. O tipo de disfonia também foi associado positivamente com o abandono da terapia, sendo que pacientes com disfonia organofuncional tiveram 2,34 mais chances de abandonarem o tratamento. O modelo apresentou boa qualidade de ajuste avaliada pelo teste de Hosmer-Lemeshow $(\mathrm{p}=0,399)$.

Tabela 3. Resultado da regressão logística binária multivariada, "alta fonoaudiológica" como categoria de referência

\begin{tabular}{|c|c|c|}
\hline \multirow{2}{*}{ Fatores } & \multicolumn{2}{|c|}{ Abandono } \\
\hline & Valor de $p$ & OR (IC95\%) \\
\hline \multicolumn{3}{|l|}{ Faltas } \\
\hline Até 2 Faltas & & 1,0 \\
\hline 3 ou mais faltas & $<0,000^{*}$ & $3,67(1,71-7,92)$ \\
\hline \multicolumn{3}{|l|}{ Tipo de disfonia } \\
\hline Funcional & & 1,0 \\
\hline Organofuncional & $0,043^{*}$ & $2,34(1,0-5,49)$ \\
\hline
\end{tabular}

* Valores significativos $(p \leq 0,05)$

Legenda: $\mathrm{OR}=$ odds ratio

\section{DISCUSSÃO}

A baixa adesão a tratamentos é um problema de ordem mundial. Nos países desenvolvidos, a adesão aos tratamentos de longa duração, na população geral, é em torno de $50 \%$, valores superiores os observados em países em desenvolvimento ${ }^{(9)}$.

$\mathrm{O}$ abandono do tratamento pode atingir cerca de $65 \%$ dos pacientes $^{(13)}$. Entretanto, no presente estudo, de acordo com os dados dos prontuários, encontrou-se maior proporção de pacientes que receberam alta quando comparados aos pacientes que abandonaram o tratamento. Não seria imprudente supor que a menor proporção de abandono tenha relação com o perfil dos pacientes estudados, uma vez que eles dependem da voz para 
exercer suas atividades profissionais. Sabe-se que a disfonia pode acarretar prejuízos na carreira ${ }^{(2,4)}$, levando a crer que a maior adesão das professoras ao tratamento esteja relacionada aos riscos de prejuízos profissionais.

A faixa etária encontrada foi compatível com a média de 42 anos ( $\mathrm{DP}=8$ ) descrita em um artigo anterior, no qual também não se verificou associação entre a idade e a adesão ao tratamento fonoaudiológico ${ }^{(2)}$. O número de filhos interfere em vários eventos e situações de saúde no grupo das mulheres ${ }^{(17)}$, mas não se encontrou associação com a adesão ao tratamento fonoaudiológico. Na literatura investigada, não foram identificados estudos que permitissem comparação quanto a esse efeito.

Quanto ao número de sessões programadas, a média de 15 sessões para ambos os grupos (Tabela 1) diverge de um estudo $^{(13)}$ que descreveu uma duração média de 5 a 5,6 sessões para a terapia de voz. Tratando-se de um hospital escola, com períodos de descontinuidade no acompanhamento ambulatorial é possível haver prolongamento do tratamento, incluindo um número superior de sessões. Ademais, o tratamento fonoterápico difere de um serviço a outro, podendo abarcar diferentes procedimentos e número de sessões. Contudo, vale mencionar a ausência de associação entre o número de sessões da fonoterapia e a adesão ao tratamento, conforme citado na literatura ${ }^{(13)}$.

As distâncias entre a residência e o local de trabalho e entre a residência e o serviço fonoaudiológico não foram associadas ao abandono ( $\mathrm{p}=0,228 \mathrm{e} \mathrm{p}=0,405$ respectivamente). Apesar da dificuldade de acesso ser mencionada entre os entraves à continuidade da terapia fonoaudiológica ${ }^{(10)}$, não foram encontrados outros estudos que focalizassem tal problemática.

No que se refere ao número de ausências às sessões, os resultados indicaram que pacientes com três ou mais faltas apresentaram 3,67 mais chances de abandonar o tratamento $(\mathrm{p}<0,000)$ (Tabela 3). A literatura não confirma a relação direta entre o número de ausências e a adesão ao tratamento fonoaudiológico. Entretanto, aponta que o número de sessões realizadas consiste no mais forte preditor para a conclusão da terapia de $\operatorname{voz}^{(13)}$. Cada nova sessão realizada (após a avaliação vocal) resultou em uma probabilidade 1,6 vezes maior de completar o tratamento vocal. Ao se considerar que o não comparecimento às sessões pode estar associado a fatores individuais, tais como o nível de motivação do paciente para com a terapia, torna-se razoável inferir sobre o seu papel na adesão. Um estudo evidencia que indivíduos que se dizem motivados para o tratamento apresentam 3,06 vezes mais chance de adesão ao tratamento não farmacológico para diabetes mellitus ${ }^{(18)}$.

Quanto ao grau de disfonia, obtido por meio da avaliação perceptivo-auditiva, os resultados são concordantes com a literatura. Não se observou associação entre o grau da disfonia e a adesão à terapia vocal ${ }^{(14)}$. Portanto, o grau do desvio vocal não parece influenciar a motivação do paciente em dar continuidade à terapia e, consequentemente, concluir o tratamento fonoaudiológico.

O tipo de disfonia também foi associado positivamente ao abandono da terapia (Tabela 3 ), sendo que os pacientes com disfonia organofuncional tiveram 2,34 mais chances de abandonar o tratamento. Considerando-se que a disfonia organofuncional corresponde à evolução clínica da disfonia funcional ${ }^{(15)}$, pode-se supor que as professoras com esse tipo de disfonia levaram mais tempo para procurar por ajuda profissional. A literatura aponta que a autoavaliação vocal de professores evidencia a satisfação da maioria dos deles quanto à própria voz, o que alude para a dificuldade desses profissionais em autoavaliar sua voz e em perceber alterações vocais ${ }^{(19)}$. É consenso que os fatores centrados na própria doença, tais como percepção do paciente acerca de seu problema de saúde e estratégias para lidar com a doença e seus sintomas e impacto na qualidade de vida são capazes de influenciar não apenas a procura por atendimento, mas também o processo de adesão ao tratamento proposto $^{(12,20)}$. Dessa forma, tal resultado pode estar associado à dificuldade de percepção do problema vocal e ao comportamento de resistência em admitir a disfonia ou em procurar por ajuda especializada.

Foi inesperado o resultado que indica ausência de associação com a renda familiar, haja vista os relatos dos pacientes sobre a influência das condições financeiras sobre o abandono da terapia fonoaudiológica ${ }^{(21)}$. Tal resultado é díspar ao de um estudo $^{(18)}$ em que se analisaram pacientes com diagnóstico de diabetes mellitus. Seriam profícuas futuras pesquisas visando esclarecer as relações entre renda e adesão.

A escolaridade também não se mostrou associada ao abandono à terapia de voz $(\mathrm{p}=0,370)$. A literatura aponta para resultados divergentes no tocante à relação escolaridade-adesão. Em estudo anterior, o nível universitário foi associado à adesão a dietas alimentares saudáveis ${ }^{(22)}$. Por outro lado, em outro trabalho não houve associação entre nível de escolaridade e adesão ao tratamento com terapia antirretroviral ${ }^{(23)}$.

O IVS visa dimensionar o acesso da população a cinco "Dimensões da Cidadania", consideradas como essenciais para se alcançá-la: ambiental, cultural, econômica, jurídica e segurança de sobrevivência ${ }^{(24)}$. As "Dimensões da Cidadania" são compostas por variáveis, que por sua vez compõem-se de indicadores, ou seja, dados numéricos a partir dos quais se calcula o IVS. O cálculo é elaborado por meio de sucessivas agregações feitas por meio de médias aritméticas ponderadas e seu valor final varia entre zero e um. Como o IVS expressa um atributo negativo, a vulnerabilidade social de uma população será maior quanto maior for seu valor, ou seja, quanto maior for o valor do IVS, mais vulnerável à exclusão social uma população estará sujeita ${ }^{(24)}$. Em suma, tem-se que o IVS combina diferentes variáveis em um indicador cuja finalidade é sintetizar informações relevantes que traduzem as desigualdades intraurbanas e áreas de risco social ${ }^{(18)}$. A maioria dos sujeitos pesquisados apresentou valores de IVS entre 0,5 a $0,63(39,1 \%)$ ou acima de $0,63(34,8 \%)$ (Tabela 2$)$, porém, não se verificou associação entre o IVS (da região da escola onde a paciente trabalhava) e o desfecho pesquisado. Tal resultado diverge dos achados de um estudo ${ }^{(18)}$, no qual moradores de áreas de elevado risco social apresentam maior chance de não adesão ao tratamento focalizado.

A adesão ao tratamento constitui um fenômeno complexo e multifatorial. Pesquisas futuras poderão contribuir na elucidação do papel de diferentes fatores e dimensões a fim de fortalecer estratégias estimuladoras da adesão ao tratamento fonoaudiológico para a disfonia em professoras. 
A terapia vocal abrangente é eficaz para aprimorar o desempenho vocal em adultos com disfonia. Diante dos achados, suscita-se o interesse em desenvolver estratégias que influenciem positivamente no quadro vocal dos docentes, entre elas melhorar as condições de trabalho e favorecer a qualidade de vida destes profissionais. Os resultados obtidos viabilizaram a reflexão sobre os fatores passíveis de influenciar processo dinâmico que caracteriza a adesão ao tratamento fonoaudiológico. Espera-se ter agregado conhecimentos para o delineamento de futuros estudos e ter contribuído para melhor abordagem dos pacientes durante o tratamento.

\section{CONCLUSÃO}

A análise de 135 prontuários de professoras atendidas no AV-UFMG em que o estudo foi realizado, no período de agosto de 2007 a dezembro de 2009 revelou que a maioria dos pacientes aderiu ao tratamento fonoaudiológico para disfonia. O número de ausências às sessões, fator relacionado ao tratamento, e a disfonia do tipo organofuncional, aspecto referente ao quadro clínico, mostraram-se associados ao abandono da terapia de voz.

\section{AGRADECIMENTOS}

À Fundação de Amparo à Pesquisa do estado de Minas Gerais - FAPEMIG (01474-10) pelos recursos financiados para a execução da pesquisa.

* LRS, LA, LCT, IB, AAA e ACCG participaram da elaboração deste estudo. Todos ajudaram a construir e desenvolver a pesquisa, desde a revisão de literatura, análise dos resultados, discussão e conclusões. LRS e LA colaboraram com a coleta dos dados.

\section{REFERÊNCIAS}

1.Roy N, Weinrich B, Gray SD, Tanner K, Stemple JC, Sapienza CM. Three treatment for teachers with voice disorders: a randomized clinical trial. J Speech Lang Hear Res. 2003;46(3):670-88.

2.de Medeiros AM, Barreto SM, Assunção AA. Voice disorder (dysphonia) in public school female teachers working in Belo Horizonte: prevalence and associated factors. J Voice. 2008;22(6):676-87.

3.Unterbrink T, Zimmermann L, Pfeifer R, Wirsching M, Brähler E, Bauer J. Parameters influencing health variables in a sample of 949 German teachers. Int Arch Occup Environ Health. 2008;82(1):117-23.

4.Roy N, Merrill RM, Thibeault S, Parsa RA, Gray SD, Smith EM. Prevalence of voice disorders in teachers and the general population. J Speech Lang Hear Res. 2004;47(2):281-93.
5.Rogerson J, Dodd B. Is there an effect of dysphonic teachers' voices on children's processing of spoken language? J Voice. 2005;19(1):47-60.

6.Ruotsalainen J, Sellman J, Lehto L, Verbeek J. Systematic review of the treatment of functional dysphonia and prevention of voice disorders. Otolaryngol Head Neck Surg. 2008;138(5):557-65.

7.Gama ACC, Bicalho VS, Valentim AF, Bassi IB, Teixeira LC, Assunção AA. Adesão a orientações fonoaudiológicas após a alta do tratamento vocal em docentes: estudo prospectivo. Rev CEFAC. 2012;14(4):714-20.

8.Jardim R, Barreto SM, Assunção AA. Condições de trabalho, qualidade de vida e disfonia entre docentes. Cad Saúde Pública. 2007;23(10):2439-61.

9.World Health Organization (WHO). Adherence to long-therm therapies evidence for action. WHO Library Cataloguing-in-Publication Data. 2003.

10. Portone C, Johns MM, Hapner ER. A review of patient adherence to the recommendation for voice therapy. J Voice. 2008;22(2):192-6.

11. van Leer E, Connor NP. Patient perceptions of voice therapy adherence. J Voice. 2010;24(4):458-69.

12. Silveira LMC, Ribeiro VMB. Grupo de adesão ao tratamento: espaço de "ensinagem" para profissionais de saúde e pacientes. Interface Comunic., Saúde, Educ. 2005;9(16):91-104.

13. Portone-Maira C, Wise JC, Johns MM, Hapner EE. Differences in temporal variables between voice therapy completers and dropouts. J Voice. 2011;25(1):62-6

14. Hapner E, Portone-Maira C, Johns MM. A study of voice therapy dropout. J Voice. 2009;23(3):337-40.

15. Behlau M, Azevedo R, Pontes P. Conceito de voz normal e classificação das disfonias. In: Behlau M. Voz: O livro do especialista. Rio de Janeiro: Revinter; 2001.p. 53-79.

16.Hirano M. Clinical examination of voice. New York: Springer Verlag. 1981.

17. Rocha LE, Debert-Ribeiro M. Trabalho, saúde e gênero: estudo comparativo sobre analistas de sistemas. Rev Saúde Pública. 2001;35(6):539-47.

18. Assunção TS, Ursine PGS. Estudo de fatores associados à adesão ao tratamento não farmacológico em portadores de diabetes mellitus assistidos pelo Programa Saúde da Família, Ventosa, Belo Horizonte. Ciênc Saúde Coletiva. 2008;13(2):2189-97.

19. Penteado RZ, Pereira IM. Qualidade de vida e saúdevocal de professores. Rev Saúde Pública. 2007;41(2):236-43.

20. Leite SN, Vasconcellos MPC. Adesão à terapêutica medicamentosa: elementos para a discussão de conceitos e pressupostos adotados na literatura. Ciênc saúde colet. 2003;8(3):775-82.

21. Esteves D, Silva MR. Estudo de adesão e abandono dos respiradores orais encaminhados para fonoterapia. São Paulo: CEFAC, 2005. Disponível em: http://www.cefac.br/publicar/arquivos/SP_adesao_e_abandono_de_ RO_na_fonoterapia.pdf

22. Martin KA, Bowen DJ, Dunbar-Jacob J, Perri MG. Who will adhere? Key issues in the study and prediction of adherence in randomized controlled trials. Control Clin Trials. 2000;21(5):195-9.

23. Colombrini MR, Coleta MF, Lopes MH. Fatores de risco para a não adesão ao tratamento com terapia antiretroviral altamente eficaz. Rev Esc Enferm USP. 2008;42(3):490-5.

24. Nahas MI. Bases teóricas, metodologia de elaboração e aplicabilidade de indicadores intra-urbanos na gestão municipal da qualidade de vida urbana em grandes cidades: o caso de Belo Horizonte. [Tese de Doutorado] São Carlos: Universidade Federal de São Carlos; 2002. 